\title{
Application of Image Analytics for Disaster Response in Smart Cities
}

\author{
Neha Chaudhuri \\ Indian Institute of Management Calcutta \\ nehac16@iimcal.ac.in
}

\author{
Indranil Bose \\ Indian Institute of Management Calcutta \\ bose@iimcal.ac.in
}

\begin{abstract}
Post-disaster, city planners need to effectively plan response activities and assign rescue teams to specific disaster zones quickly. We address the problem of lack of accurate information of the disaster zones and existence of human survivors in debris using image analytics from smart city data. Innovative usage of smart city infrastructure is proposed as a potential solution to this issue. We collected images from earthquake-hit smart urban environments and implemented a CNN model for classification of these images to identify human body parts out of the debris. TensorFlow backend (using Keras) was utilized for this classification. We were able to achieve $83.2 \%$ accuracy from our model. The novel application of image data from smart city infrastructure and the resultant findings from our model has significant implications for effective disaster response operations, especially in smart cities.
\end{abstract}

\section{Introduction}

Smart cities are an attempt to improve the effectiveness of urban services and enhance the quality of life. They include smart buildings, smart healthcare, education, transportation and other services that leverage information and communication technologies (ICT) [1]. These urbanized areas are built upon vast networks of sensors, cameras, and other connected smart devices, capable of capturing rich, real-time information from every nook and corner of the city continuously. This, combined with the growing need to effectively manage and analyze such heterogeneous and unstructured data streams for help in decision-making, have prompted regulators and other stakeholders to build strategic partnerships with various private vendors as well as academicians to gain rich insights from the collected data. Of the various analytics techniques available, researchers have demonstrated effective usage of machine learning (ML) techniques for solving various developmental issues, in such smart city environments, like traffic management [2], healthcare
[3], crime rates[4], and energy consumption [5]. However, an extensive review of the extant literature reveals that although there has been a focus on solutions for issues faced by traditional urban settlements, there exists an apparent lack of implementation of advanced data analytics capabilities from the massive amount of data generated from these cities for continuity planning activities like disaster management.

The domain of disaster management is focused "to mitigate, prepare for, respond to and recover from disasters with the ultimate goal to save lives, property and the environment" [6]. To ensure effective management during disaster response stage, timeliness and participation of human hands from multiple, geographically distributed organizations and their communication are critical [7]. Hence, information management plays a crucial role in disaster response operations involving strategic planning to ensure timely and effective prioritization and allocation of resources amongst discrete response activities. A report by the US-based National Research Council (U.S.) has asserted that IT has unrealized potential to improve how communities, individuals handle disasters [8]. However, the existence of incompatible systems and technologies due to lack of universal protocols for data sharing and management in these cities creates issues for effective planning. The lack of an integrated and ubiquitous system compels decision makers during disasters to often choose a sub-optimal decision based primarily on heuristics [9]. Hristidis et al. [9] highlighted these concerns of decision makers, especially for disaster management. They have particularly asserted the need to integrate discrete data sources and resources in a collaborative and timely manner, and the need to collect updated information from these distributed sources through one integrated information system of the city [9]. This combined with the adoption of advanced data analytics will aid effective decision making in disaster situations.

However, the limited amount of studies in this domain such as those by Asimakopoulou and Bessis [6] or Alazawi et al. [10] highlight the lack of focus on utilizing the capabilities of data analytics, holistically, for disaster management as a 'smart service' in a smart 
city. In response to this gap in the extant literature, our current study attempts to examine the effectiveness of a specialized ML technique for disaster management in smart cities. We state our precise research question as:

RQ: How can Image Analytics be used for disaster management in smart city context?

The effectiveness of disaster response operations is primarily determined by the timeliness in identifying and prioritizing disaster zones. With existing infrastructure of cameras throughout smart cities, visual data is a rich source of information to aid such decisions. Lack of such infrastructure in conventional urban environments dissuades any possibility of capturing such data during disasters. Although many of these devices might be rendered ineffective during disasters even in smart cities, the limited ones that survive, provide a quick glimpse of the disaster situation and hence aid such decision making. Image analytics techniques based on ML can be utilized for such prioritization of rescue efforts. Inspired by this novel application of image analytics, our research utilizes convolutional neural networks for classification of huge amounts of image data with the help of Keras with TensorFlow as backend. Our model classifies images with a high degree of accuracy of the existence of human survivors in debris from photographs of disaster zones. Identification of survivors from partial body parts visible in images is challenging to decipher from a huge dataset of images provided by smart city infrastructure[11]. Hence, classification of these images through cloud-based ML techniques improves the efficiency of search operations for timely disaster response.

This study makes several key contributions. By combining a relatively less used but powerful method on the less utilized visual content of big data of smart cities in a novel application and presenting a deep learning-based study, we illustrate the potential of ML techniques for such contexts. Apart from the novelty of application, this method also highlights a novel and efficient technique for ML classification by utilizing backend from Google which is a global market leader in ML. Further, our comprehensive review of image data analytics in the domain of disaster management with particular focus on smart cities serves as a compendium of knowledge on the topic for future studies. With a focus on generalizability, the insights from this study will aid practitioners (disaster managers and rescue teams) and simultaneously expand the knowledge base on image analytics of big data.

\section{Background}

\subsection{Smart Cities}

The term 'smart city' has been used since 2005 by global technology firms to refer to use of complex information and communication technologies (ICT) to integrate the operation of urban infrastructure and services such as buildings, transportation, electrical and water distribution, and public safety [12]. This concept is seen as an opportunity for urban development and simultaneous improvement of both the city's livability and its local economy. An example of a smart city would be Singapore, which was ranked as the top performer in a global smart city ranking by Juniper Research in 2018 [13].

However, owing to differences in working definitions due to varying perspectives and advances in technologies over time, a smart city has historical predecessors such as intelligent city [14], knowledge city [15], ubiquitous city [16] and digital city [17]. The significant difference between a smart city and its predecessors lies in the former's inclusion of human capital component as one of its three key elements. While the earlier versions emphasized the technological dimension only, a smart city focuses on the balanced combination of technological, institutional and human dimensions [18].

2.1.1. Classification of smart city services. Smart cities enact distinct roles to positively impact diverse facets of citizens' lives. The classification of these varied smart city services studied in literature is described in Table 1. The table also reveals a distinct lack of focus on utilizing the power of data analytics for disaster management as a 'smart service' in a smart city context.

\subsection{Disaster Management}

Disaster management is comprised of four major phases: mitigation and preparedness (pre-disaster), response (during a disaster), and recovery (postdisaster) [19]. These phases are mutually exclusive and highly interconnected [19].

Extant literature has primarily focused on specific phases of disaster management. There has been limited research on pre-disaster phases including preparedness and mitigation. However, whatever little work has been done, the focus has been primarily on the development of extensive theoretical frameworks [20]. 
Table 1: Literature review for services in a smart city

\begin{tabular}{|c|c|c|}
\hline Smart city services category [21] & Author [Year] & Summary of work \\
\hline Smart Urban Management & $\begin{array}{ll}- & \text { Anthopoulos et al. [22] } \\
\text { - } & \text { Rathore et al. [23] }\end{array}$ & $\begin{array}{l}\text { - Interrelation of physical and digital space } \\
\text { of smart city with tangible measurement } \\
\text { - combined Internet of Things-based system } \\
\text { for smart city development and urban } \\
\text { planning using Big Data analytics }\end{array}$ \\
\hline Smart Transportation & $\begin{array}{l}\text { - } \quad \text { Vlahogianni et al. [2] } \\
\text { - } \quad \text { Barba et al. [24] }\end{array}$ & $\begin{array}{l}\text { - Real-time parking prediction system for } \\
\text { smart cities } \\
\text { - Framework to transmit information about } \\
\text { traffic conditions to help vehicles take } \\
\text { adequate decisions }\end{array}$ \\
\hline Public Health, Medical Care \& Welfare & Hassan et al. [3] & $\begin{array}{l}\text { - Cloud-Assisted Internet of Things (IoT) } \\
\text { Framework for Pervasive Healthcare in } \\
\text { Smart City Environment } \\
\text { - } \quad \text { Voice pathology detection paradigm }\end{array}$ \\
\hline $\begin{array}{l}\text { Facilities \& Environment } \\
\text { (included waste management, energy, } \\
\text { water, and sanitation management) }\end{array}$ & $\begin{array}{ll}\text { - } & \text { Kanchev et al. [5] } \\
\text { - } & \text { Srikantha et al. [26] }\end{array}$ & $\begin{array}{ll}\text { - } & \text { Energy management for smart grid } \\
\text { applications } & \\
\text { - } & \text { IoT-enabled waste management }\end{array}$ \\
\hline Culture Tourism \& Sports & $\begin{array}{ll}\text { - } & \text { Gretzel et al. [27] } \\
\text { - } & \text { Brandt et al. [28] }\end{array}$ & $\begin{array}{l}\text { - } \quad \text { Smart tourism ecosystem } \\
\text { - Spatial and semantic analysis of social } \\
\text { media messages for smart tourism } \\
\text { ecosystems }\end{array}$ \\
\hline $\begin{array}{l}\text { Education } \\
\text { (remote education service) }\end{array}$ & Hughes et al. [29] & $\begin{array}{l}\text { Remote human surrogates and 'avatars' to } \\
\text { teach in virtual environments }\end{array}$ \\
\hline Crime \& Disaster Prevention & Pan et al. [4] & Research issues in trace analysis for crime \\
\hline
\end{tabular}

However, research on disaster response has been more diverse. Various studies have investigated past disasters and suggested effective solutions through the efficient use of technologies and social media [30], or crowdsourcing for updated geographic information [31]. Of the limited empirical studies on post-disaster phase, Kunreuther et al. [32] examined the financial allocation for disasters. Such empirical studies have the potential to extend the theoretical developments in this domain leading to the development of a robust knowledge base for future research.

\subsection{Disaster Management in Smart cities}

The data generated from continuous streams from sensors, cameras, and other smart city infrastructure have the potential to improve disaster management operations. There have been various studies aimed at utilizing the interconnected modules of various sensors and cameras of smart cities for disaster management and monitoring of response activities [33]. Asimakopoulou and Bessis discussed the role of crowdsourcing in the context of smart buildings to support effective disaster management [6]. Alazawi et al. discussed the importance of building frameworks for smart city-based disaster management context. Other notable studies in this domain have also proposed a framework for implementing intelligent transportation systems including vehicular ad hoc networks (VANETs) and cloud computing technologies [10], and smartphonebased mobile disaster management system for optimal geographical routing for volunteers and resources under limitations of time [34]. However, to the best of the author's knowledge, quantitative studies utilizing machine learning to investigate the potential of the visual content of the captured big data in smart cities effective disaster search and response were lacking. We aim to position our study in response to this gap in the extant literature.

\section{Research Design}

\subsection{Image Analytics with Deep Learning}

With most of the smart city data being unstructured in the form of sensor data and visuals (be it images or video), image classification and subsequent analysis have the potential to provide efficient solutions to accelerate decision-making processes. This has prompted research to use machine learning algorithms for text as well as image analytics. Image analytics not only finds application in creating product and marketing solutions for business; but also, in defense and security as well as healthcare. For example, defense agencies have been reported to have been extensively spending on storage and analysis of signal data, visuals, and fullmotion videos and having been using image and video 
analytics successfully. They have been using drones to capture intelligence via video and image surveillance to mitigate risks of direct intervention both in urban and rural landscapes.

In line with practical applications of image analytics, this article highlights usage of these techniques for disaster management. Image analysis is defined as the "algorithmic extraction and logical analysis of information found in image data using digital image processing" [35]. It is an extension of text analysis features used on visual content. Before analyzing images, the geometric encoding for each raster image (sequence of pixels with discrete numerical values for the color of an image) is transformed into constructs depicting physical features and objects. Though unsupervised machine learning algorithms have been traditionally used for image content analysis in areas such as bioinformatics, oceanography, computer vision; these methods suffer from poor performance. Thus, we use deep learning methods (a subfield of machine learning). Deep learning extracts hierarchical representations from large-scale data (e.g., images) with multiple layers of non-linear transformations. It extracts different levels of information ingrained in input data by designing the layer depth and width, and intricately selecting features that are useful for learning tasks.

\subsection{Convolutional Neural Networks}

There are several classes of deep learning models such as Deep Belief Networks (DBN) [36], Convolutional Neural Networks (CNN) [37], Deep Boltzmann Machines (DBM) [38], Stacked Denoising Auto-Encoders [39], etc. The widely used among these models is the Convolutional Neural Network which finds applications in image classification [40], object tracking [41], pedestrian detection [42], image labeling [43], and speech recognition [44] amongst others. Its huge adoption is primarily due to the ease of training of the model since it estimates fewer parameters as compared to the other deep learning models. This has been possible because the neurons of a $\mathrm{CNN}$ in one layer are not necessarily connected to all the neurons in the layer before it. For more details, refer [37]. Moreover, these layers are arranged in three dimensions, which allows images to be provided as inputs for advanced analytics.

\subsection{TensorFlow}

TensorFlow is an open source software library designed by researchers from Google Brain team for high-performance numerical computation. It is highly efficient in applications requiring the building of large and flexible deep learning models. Additionally, it offers:

i. Threading and queueing for computing tensors asynchronously. It expedites computations by allowing parallel processing.

ii. Greater control and easier operations on weights or gradients in the developed CNN model.

iii. Faster compile time than other competing open source frameworks.

TensorFlow has the highest adoption both in industry and academic research community primarily due to its efficient utilization of computing resources especially GPUs. These features improve the processing speed of image data for use in this study. As discussed earlier, timeliness and accuracy are very crucial for disaster response planning. That combined with the cloud-based infrastructure of TensorFlow, allows for resource offloading to offshore servers while simultaneously providing quicker processing. These capabilities have guided our choice of TensorFlow library through the $\mathrm{R}$ interface using the high-level Keras API.

3.3.1. Keras It is a neural network API which interfaces with advanced deep learning and implementation frameworks like TensorFlow and Theano. It allows for rapid prototyping of deep learning models with minimal lines of code. To gain an understanding of its popularity backed by its rich feature set, we should identify its application in academic research. In that parameter, Keras is ranked second in terms of mentions in scientific papers uploaded to the preprint server at arXiv.org.

Our adoption of Keras for this study is based on its ease of interfacing with TensorFlow, flexibility, and control over the proposed deep learning model. Specifically, we have used Keras built on TensorFlow for analysis because of its following features:

i. It supports both convolutional networks and recurrent networks, as well as combinations of both.

ii. It supports several network architectures such as the multi-input or multi-output models, layer sharing, model sharing.

iii. It allows enhanced ease of use, modularity, extensibility and increased flexibility.

\section{Data Processing}

\subsection{Data Collection}

With an explicit focus on proposing innovative solutions for effective decision making for disaster response using machine learning techniques, we adopt 
an innovative application of images from disaster (specifically earthquake-hit) zones for this study. This was used as input to our proposed CNN model. Our initial image dataset was drawn from the National Hazards Image Database of the National Centers for Environmental Information (www.ngdc.noaa.gov/haz ardimages/). With focus on disasters in smart cities, we collected images of earthquake-hit regions of Tohoku, Japan (2011) and Bologna, Italy (2012) from that dataset. Both of these were smart cities when disaster struck, but the lack of application of image analytics for disaster management resulted in a small dataset. We then compared images of smart cities hit by earthquakes with those of conventional urban spaces affected by earthquakes. We also supplanted our initial dataset with other images from the Google Image database (Creative Common images were used). This approach was in line with similar studies which have used similar social media images for analytics in the domain of disaster management [46]. Images were collected using the filter 'Labeled for non-commercial reuse with modification.' The search was conducted using Google search, e.g., by searching for 'people in debris earthquake Bologna 2012'. We collected all images published within two months of each incident by restricting the timeline using the 'custom time range' filter.

\subsection{Data Preparation}

The image dataset went through a simple annotation process to decide whether each image was related to the chosen incidents. For this, two external researchers, who were domain experts, independently evaluated all the images manually by checking their sources and appropriateness for our study. The researchers further pruned the dataset by removing images which had captured relief workers searching and rescuing trapped people. These images were not considered to be relevant for our analysis as our CNN model classifies images based on whether any human body part or whole, is found to be trapped under debris and needs to be rescued. The images would get classified based on whether such a victim is present under the debris or not, and presence of rescue workers would lead to a 'false positive.' Hence, few images had to be removed, and the remaining dataset then underwent pre-analysis/preprocessing. Following the image pre-processing steps as discussed in Caffe [47], all images were resized uniformly to ensure that they have identical size and aspect ratio. Following this, global zero-mean unit variance batch normalization was carried out to improve training stability and resistance to overfitting of image data. Additionally, common data augmentation parameters to further address the issue of overfitting of image data including rotation, translation, rescaling, flipping, shearing and stretching [37]. Hence, data augmentation was lastly performed on our dataset to add perturbed versions of the existing images to the existing dataset. At the end of data preparation stage, our final dataset comprised of 541 images.

\subsection{Data Analysis}

Image classification was performed by a convolutional neural network model. Since real-time classification is an important factor for effective disaster response, we avoided using pre-trained models, such as the VGGNet models [55], in this paper as they have been found to suffer from significantly higher training time and correspondingly higher computation time and resources [56]. Thus, we built our $\mathrm{CNN}$ model, and all computations were done using the software package $\mathrm{R}$ [48] and the R package KERAS (R interface to 'Keras') [49] with TENSORFLOW library ( $\mathrm{R}$ interface to 'TensorFlow') [50].

Following standard practice in predictive research, we evaluated the model's performance "out-of-sample" [51]. We used one set of data to train our model and another one to test it. For this, the original dataset was split by 80-20 for training and testing sets respectively. Further, since the aim of our model was to classify images based on the presence of humans trapped under debris, our dataset consisted of categorical/classifiable images. However, a CNN model is not capable of identifying classes directly which led us to the next step of modifying our classifiable dataset. We used One-Hot Encoding and assigned a new binary value for each unique class description [52]. Thus, "0" represented images without any human traces and " 1 " represented images with human body parts present.

Finally, the model was built as a sequence of four types of layers: convolutional layer (conv), pooling layer (pool), rectified linear unit layer (reLU) and fullyconnected layer (fc).

i. Input $[128 \times 128 \times 3]$ : held the raw pixel values of each image;

ii. conv layers: computed output of neurons connected to local regions of input;

iii. $\quad$ reLU layers: applied elementary activation function;

iv. pool layer: performed down-sampling along spatial dimensions;

v. classifier layer: last layer of model ('softmax' function used under cross-entropy regime) [49].

The parameter description of the layers in our $\mathrm{CNN}$ model is as shown in Table 2. 
Table 2: Parameter description of layer in CNN model

\begin{tabular}{lll}
\hline \hline Layer Type & $\begin{array}{l}\text { Memory ( in } \\
\text { Millions) }\end{array}$ & Parameters \\
\hline \hline Input & 0.049 & 0 \\
\hline conv3_64 & 1.05 & 1,728 \\
\hline pool2 & 0.24 & 0 \\
\hline conv3_128 & 0.52 & 73728 \\
\hline conv3_128 & 0.52 & 147456 \\
\hline pool2 & 0.14 & 0 \\
\hline conv3_256 & 0.27 & 294912 \\
\hline conv3_256 & 0.27 & 589824 \\
\hline pool2 & 0.07 & 0 \\
\hline conv3_512 & 0.13 & 1179648 \\
\hline conv3_512 & 0.13 & 2359296 \\
\hline pool2 & 0.03 & 0 \\
\hline fc & 0.005 & 102760448 \\
\hline Total memory: $2.2 \mathrm{M} * 4$ bytes $\sim=8.9 \mathrm{MB} /$ image \\
\hline Total parameters: $107 \mathrm{M}$ parameters \\
\hline
\end{tabular}

\section{Results}

To study whether image classification and analysis method could help classify images of disaster-affected zones based on the presence of humans trapped under debris, we built our CNN model as described in the previous section. A summary of our results are presented in Figure1(a) and Figure 1(b). The horizontal axes represent the number of 'epochs' of the model, where one epoch is equal to the number of images divided by the batch size.

The model measures performance by evaluating loss and accuracy on the training and testing sets for each epoch, giving rise to four values: training loss, testing loss, training accuracy and testing accuracy. Accuracy lies in the range of $[0,1]$ while the loss is not. This is so because loss is measured as a negative log-likelihood for errors made in training or testing sets for each image.

The plots of both losses in Figure 1(a) show a decreasing nature with each epoch and those of accuracies in Figure 1(b) display an increasing nature as expected.

\section{Discussion}

To describe the performance of the classifier model on our image dataset, we created a $2 \times 2$ confusion matrix as shown in Table 3. The four quadrants (clockwise, starting from the first left corner) represent four cases of results from the classification model, which are as follows:

i. 1st quadrant: TP (true positive) denotes the number of correctly detected images with human body parts present;

ii. 2nd quadrant: FP (false positive) denotes the number of images without human body parts that are incorrectly detected as those with human body parts;

iii. 3rd quadrant: $\mathrm{FN}$ (false negative) represents the number of images with human body parts present that are detected as those without human body parts; and

iv. 4th quadrant: TN (true negative) represents the number of correctly classified images without any human traces.

Hence, as shown in Table 3, for the final dataset of 541 images, our proposed CNN model was able to accurately classify 450 images. These accurately classified images belong to either of TP or TN cases. Whereas, FP and FN are the cases where images were

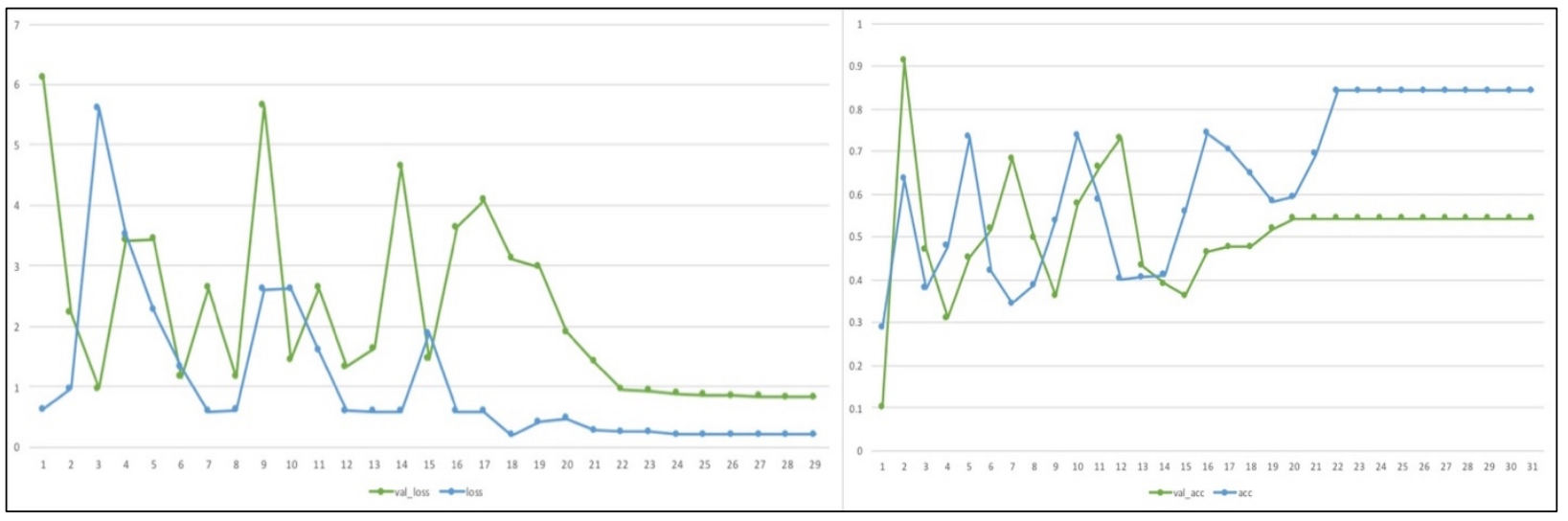

Figure 1: (a) Plot of training loss (loss) and testing loss (val_loss) (b) Plot of training accuracy (acc) and testing accuracy (val_acc) 
incorrectly classified, which provide scope to enhance the performance of the model. Thus, one of these two false cases generally needs to be minimized depending on the problem situation and its context. Since the primary aim of disaster response management is to save lives, authorities can afford to have false positive situations, but not false negatives. While false positives will increase the resources needed for disaster response, false negative might lead to loss of lives due to misclassification. False positives, in this situation, are acceptable within a limited range too.

Extending our discussion on performance, we should note that the confusion matrix is not a performance measure of a model in itself, rather performance metrics can be derived from it. Of all the available metrics to judge classification models, the performance of our image classification model was evaluated using the four commonly used metrics: precision $(\mathrm{P})$, recall $(\mathrm{R}), \mathrm{F}-$ measure $(\mathrm{F})$, and accuracy (A) [53] (defined in Equations (1)-(4)).

$$
\begin{aligned}
P & =\frac{T P}{T P+F P} \ldots \ldots \ldots \ldots \ldots \ldots \ldots \ldots \ldots \ldots \ldots \ldots \ldots \ldots \ldots \ldots \\
R & =\frac{T P}{T P+F N} \ldots \ldots \ldots \ldots \ldots \ldots \\
F & =\frac{2 P R}{P+R} \ldots \ldots \ldots \ldots \ldots
\end{aligned}
$$

where TP, TN, FP, and FN have meanings as discussed above.

The overall accuracy of our model was found to be $83.18 \%$ which is an acceptable measure for such classification models, refer [54]. Table 4 shows the prediction accuracy scores for a sample of images obtained by our model. While our model accurately identifies the three images, it is unable to decisively predict the third image. Precision signifies the proportion of images that our model correctly identified as having human body parts out of all images that had human body parts present. The precision for our model stands at $85.87 \%$. Another metric to measure the accuracy of CNN models is recall which signifies the proportion of images that actually had human body parts present and were also identified as having human body parts. The recall for our model was calculated as $82.6 \%$. While precision reveals how our model performed with respect to FP, recall reveals its performance with respect to FN. Therefore, recall is slightly more important in this context where the aim is not to classify all images correctly, but to accurately classify images that have any human body part present. Since any mistake in identifying a human body part in debris can directly be fatal to human lives and hence will make this entire exercise irrelevant for disaster response. The F-measure of our model was found to be $83.89 \%$. It is a combined metric of precision and recall.

Our findings indicate that a multi-layer convolutional neural network is adept at obtaining significant results on a large challenging dataset with supervised learning. We found that our model's performance kept on reducing by around $1.3 \%$ as we kept removing each convolutional layer. This indicated that depth was necessary for satisfactory results.

Table 3: $2 \times 2$ Confusion matrix for the $\mathrm{CNN}$ model

\begin{tabular}{llll}
\hline $\mathrm{n}=541$ & Actual “1” & Actual “0” & RowTotal \\
\hline Predicted "1" & $237 *$ & $39 * *$ & 276 \\
Predicted "0" & $52 * * *$ & $213 * * * *$ & 265 \\
\hline *True Positive **False Positive ***False Negative ****True \\
Negative \\
"0" refers to images without human traces \\
"1" refers to images with human body parts present
\end{tabular}

Also, we did not use unsupervised learning, but we expect that it will have a positive impact on results. However, usage of unsupervised learning for practical purposes has its own trade-offs. While it is expected to perform significantly well with the same amount of image data as has been used in this article, but it would be achievable at the cost of higher computational power to significantly increase the depth of the convolutional neural network.

\section{Implications}

Our findings have significant theoretical implications for usage of the huge visual smart city data for disaster management, and in particular for search and response tasks. Further, our extensive review of existing literature on the usage of unstructured smart city data (primarily textual and visual) serves as an easy reference to the various dimensions of research on this topic. We noted that this data had been analyzed primarily for developmental issues of smart cities like transportation, vehicle parking, waste, and energy management. Also, a review of applications of image classification reveals that it has mostly been studied for bioinformatics, medical imagery, drug compositions, while very few works exist for urban solutions. Hence, our innovative approach to the usage of advanced ML techniques for smart city disaster management provides new avenues for future research. Our novel application of existing visual data streams from smart city devices presents new opportunities for further research in smart city management domain. Although we have used images from earthquake-hit zones of smart urban environments, this analysis can be extended to other disasters like landslides, forest fires, etc. with some modification and further training of the CNN model.

Our study has important practical impliandeos2 for disaster management in general and smart cities 
in particular. Our demonstration of the potential of image data analytics from existing data streams for disaster management provides a novel application of smart city infrastructure. The findings of the study will be useful for smart city planners and regulators. The benefits of more accurate information of the disaster situation will be especially helpful for disaster response planning. Improvements in efficiency and effectiveness of disaster response planning also ease the stress on limited resources available immediately after. Hence, the learnings from this approach will be extensible to conventional urban infrastructures later.

Lastly, the proposed model is expected to witness a linear increase in computational time and resources required with an increase in number of layers (i.e., depth of the network) and an increase in number of input images. However, as timeliness is a major concern in disaster response activities, these challenges need to be addressed in real life situations to maintain the model's efficiency. For example, this could be done by using pooling layers which are responsible for reducing input size at each convolutional layer of CNN model.

\section{Conclusion}

Our study proposes a novel approach to disaster response specifically for smart city environments while stressing the importance of the immediate lifesaving response to rescue trapped survivors in disaster zones. Initial stages of search and response necessitate proper planning to assign the limited resources, such as geographically distributed rescue and first aid teams along with their heavy technical equipment, to disaster locations. Moreover, the search and rescue work in some locations may be challenging depending on the extent of damage to the urban structures and public services in those areas. Thus, effective response post-disaster requires accurate and updated information of the types of structures affected, the extent of damage, layout of the affected areas, potential hazards and most importantly about the number of lives trapped. This assessment is especially crucial for disaster response.

However, these disaster-hit zones are risky for rescue workers too. Hence, it is important to identify solutions to conduct search and rescue operations safely with accurate knowledge of the disaster zone. This study is an attempt to alleviate these concerns. Our focus was on requiring minimal changes to the existing smart city infrastructure by utilizing huge amounts of data already captured by the installed cameras to aid decision makers and rescue team workers. Though our proposed solution would be applicable for every disaster situation with the help of camera-equipped drones which are slowly becoming common in rescue teams. However, this technique will be difficult to implement in conventional urban environments as it would also add to the already skyrocketing costs due to damages from the disaster. As these techniques mature, conventional urban spaces might also consider equipping themselves with cameras and other appropriate infrastructure for disaster preparedness.

Thus, deep learning on the unstructured visual data of a smart city holds promise for large-scale implementation. Our study is in progress and extension of the ongoing research would be necessary before being deployed in actual disaster situations. We will be expanding our image dataset to make our model richer and operationalizable. As social media images, taken on-the-ground during disaster situations, could also provide valuable information to further enhance disaster response activities, we will be incorporating them into our future study. We will also be extending our analysis using deep convolutional networks to analyze video data streams that have the potential to provide richer information than static images.

Table 4: Sample input images and their classification by the proposed image analytics method

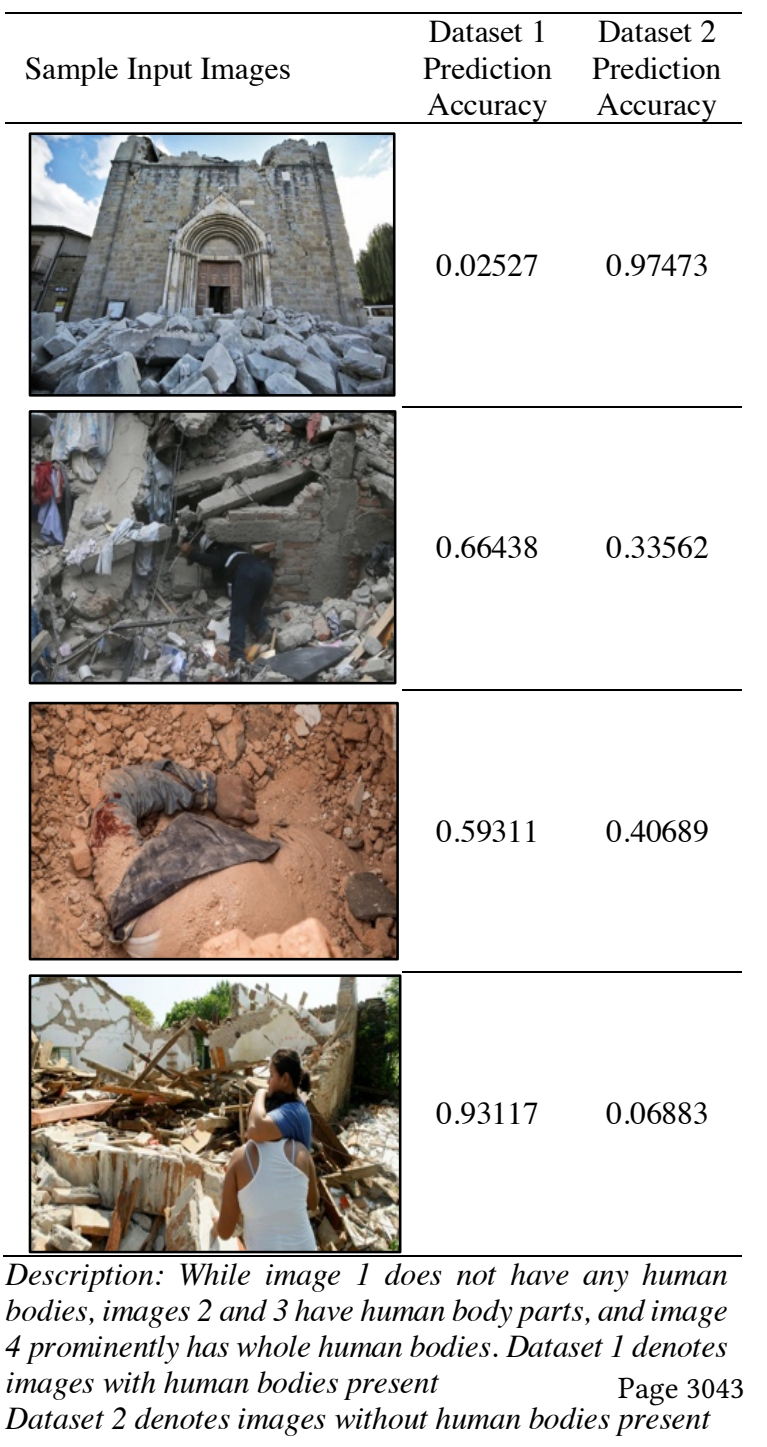




\section{References}

[1] B. Gontar, Z. Gontar, and A. Paumla, "Deployment of Smart City Concept in Poland. Selected Aspects," Manag. Organ. Syst. Res., vol. 67, pp. 39-51, 2013.

[2] E. I. Vlahogianni, K. Kepaptsoglou, V. Tsetsos, and M. G. Karlaftis, "A Real-Time Parking Prediction System for Smart Cities," J. Intell. Transp. Syst. Technol. Planning, Oper., vol. 20, no. 2, pp. 192-204, 2016.

[3] M. M. Hassan, H. S. Albakr, and H. Al-Dossari, "A Cloud-Assisted Internet of Things Framework for Pervasive Healthcare in Smart City Environment," in Proceedings of the 1st International Workshop on Emerging Multimedia Applications and Services for Smart Cities - EMASC'14, 2014, pp.9-13.

[4] G. Pan, G. Qi, W. Zhang, S. Li, Z. Wu, and L. Yang, "Trace analysis and mining for smart cities: Issues, methods, and applications," IEEE Commun. Mag., vol. 51, no. 6, pp. 120-126, 2013.

[5] H. Kanchev, D. Lu, F. Colas, V. Lazarov, and B. Francois, "Energy management and operational planning of a microgrid with a PV based active generator for smart grid applications," IEEE Trans. Ind. Electron., vol. 58, no. 10, pp. 45834592, 2011.

[6] E. Asimakopoulou and N. Bessis, "Buildings and Crowds: Forming Smart Cities for More Effective Disaster Management," in 2011 Fifth International Conference on Innovative Mobile and Internet Services in Ubiquitous Computing, 2011, pp. 229-234.

[7] J. S. S. Santiago, W. S. Manuela, M. L. L. Tan, S. K. Sañez, and A.Z. U. Tong, "Of timelines and timeliness: lessons from Typhoon Haiyan in early disaster response," Disasters, vol. 40, no. 4, pp. 644-667, 2016.

[8] National Research Council (U.S.). Committee on Using Information Technology to Enhance Disaster Management., R. R. Rao, J. Eisenberg, and $\mathrm{T}$. Schmitt, Improving disaster management: the role of IT in mitigation, preparedness, response, and recovery. National Academies Press, 2007.

[9] V. Hristidis, S.-C. Chen, T. Li, S. Luis, and Y. Deng, "Survey of data management and analysis in disaster situations," J. Syst. Softw., vol. 83, no. 10, pp. 1701-1714, 2010.

[10] Z. Alazawi, O. Alani, M. B. Abdljabar, S. Altowaijri, and R. Mehmood, "A smart disaster management system for future cities," in Proceedings of the 2014 ACM international workshop on Wireless and mobile technologies for smart cities - WiMobCity '14, 2014, pp. 1-10.

[11] C. Plagemann, V. Ganapathi, D. Koller, and S. Thrun, "Real-time identification and localization of body parts from depth images," in Proceedings - IEEE International Conference on Robotics and Automation, 2010, pp. 3108-3113.

[12] I. a. Harrison, C. and Donnelly, "A Theory of Smart Cities," Proc. 55th Annu. Meet. ISSS 2011, Hull, UK, no. Proceedings of the 55th Annual Meeting of the ISSS, pp. 1-15, 2011.
[13] Juniper Research, "Smart Cities - What's in it for Citizens?," White Pap., 2018.

[14] R. G. Hollands, "Will the real smart city please stand up? Intelligent, progressive or entrepreneurial?," City, vol. 12, no. 3, pp. 303320, 2008.

[15] C. McFarlane and O. Söderström, “On alternative smart cities: From a technologyintensive to a knowledge-intensive smart urbanism," City, vol. 21, no. 3-4, pp. 312-328, 2017.

[16] O. Kwon and J. Kim, "A Methodology of Identifying Ubiquitous Smart Services for UCity Development," in UIC 2007, 2007, no. LNCS 4611, pp. 143-152.

[17] M. J. Moon, "The Evolution of E-Government among Municipalities: Rhetoric or Reality?," Public Adm. Rev., vol. 62, no. 4, pp. 424-433, 2002.

[18] H. Lindskog, "Smart communities initiatives," Proc. 3rd ISOneWorld Conf., no. April, p. 16, 2004.

[19] V. Hwacha, "Canada's experience in developing a national disaster mitigation strategy: A deliberative dialogue approach," Mitigation and Adaptation Strategies for Global Change, vol. 10, no. 3. pp. 507-523, 2005.

[20] A. Maskrey, Disaster mitigation : a community based approach. Oxfam, 1989.

[21] J. Lee and H. Lee, "Developing and validating a citizen-centric typology for smart city services," Gov. Inf. Q., vol. 31, no. SUPPL.1, 2014.

[22] L. G. Anthopoulos and A. Vakali, "Urban Planning and Smart Cities: Interrelations and Reciprocities," in The Future Internet. FIA 2012. Lecture Notes in Computer Science, 1st ed., J. D. Federico Alvarez, and T. Z. Petra Turkama, Eds. Springer-Verlag Berlin Heidelberg, 2012, pp. 178-189.

[23] M. M. Rathore, A. Ahmad, A. Paul, and S. Rho, "Urban planning and building smart cities based on the Internet of Things using Big Data analytics," Comput. Networks, vol. 101, pp. 6380,2016

[24] C. T. Barba, M. Á. Mateos, P. R. Soto, A. M. Mezher, and M. A. Igartua, "Smart city for VANETs using warning messages, traffic statistics and intelligent traffic lights," in IEEE Intelligent Vehicles Symposium, Proceedings, 2012, pp. 902-907.

[25] M. S. Hossain, G. Muhammad, and A. Alamri, "Smart healthcare monitoring: a voice pathology detection paradigm for smart cities," Multimedia Systems, pp. 1-11, 2017.

[26] N. Srikantha, K. Moinuddin, L. K. S, and A. Narayana, "Waste Management in IoT-Enabled Smart Cities: A Survey," Int. J. Eng. Comput. Sci., vol. 6, no. 6, pp. 2319-7242, 2017.

[27] U. Gretzel, H. Werthner, C. Koo, and C. Lamsfus, "Conceptual foundations for understanding smart tourism ecosystems," Comput. Human Behav., vol. 50, pp. 558-563, 2015.

[28] T. Brandt, J. Bendler, and D. Neumann, "Social media analytics and value creation in urban smart tourism ecosystems," Inf. Manag., vol. 54,980364,4 pp. 703-713, 2017. 
[29] C. E. Hughes and C. E., "Human Surrogates: Remote Presence for Collaboration and Education in Smart Cities," in Proceedings of the 1st International Workshop on Emerging Multimedia Applications and Services for Smart Cities - EMASC'14, 2014, pp. 1-2.

[30] B. Ross, T. Potthoff, T. A. Majchrzak, N. R. Chakraborty, B. Lazreg, and S. Stieglitz, "The Diffusion of Crisis-Related Communication on Social Media: An Empirical Analysis of Facebook Reactions," Proc. 51st Hawaii Int. Conf. Inf. Syst., pp. 2525-2534, 2018.

[31] M. F. Goodchild and J. A. Glennon, "Crowdsourcing geographic information for disaster response: A research frontier," Int. J. Digit. Earth, vol. 3, no. 3, pp. 231-241, 2010.

[32] H. C. Kunreuther and J. Linnerooth-Bayer, "The financial management of catastrophic flood risks in emerging-economy countries," Risk Analysis, vol. 23, no. 3. pp. 627-639, 2003.

[33] P. Sakhardande, S. Hanagal, and S. Kulkarni, "Design of disaster management system using IoT based interconnected network with smart city monitoring," in 2016 International Conference on Internet of Things and Applications, IOTA 2016, 2016, pp. 185-190.

[34] J. T. B. Fajardo and C. M. Oppus, "A mobile disaster management system using the Android technology," WSEAS Trans. Commun., vol. 9, no. 6, pp. 343-353, 2010.

[35] P. Raj and A. C. Raman, Handbook of research on cloud and fog computing infrastructures for data science.

[36] R. Salakhutdinov and G. Hinton, "Deep belief networks," Scholarpedia, no. April, pp. 4-5, 2009.

[37] A. Krizhevsky, I. Sutskever, and G. E. Hinton, "ImageNet Classification with Deep Convolutional Neural Networks," ImageNet Classif. with Deep Convolutional Neural Networks, pp. 1097-1105, 2012.

[38] R. Salakhutdinov and G. Hinton, "Deep Boltzmann Machines," in AISTATS, 2009, vol. 1, no. 3, pp. 448-455.

[39] P. Vincent and H. Larochelle, "Stacked Denoising Autoencoders: Learning Useful Representations in a Deep Network with a Local Denoising Criterion Pierre-Antoine Manzagol," J. Mach. Learn. Res., vol. 11, pp. 3371-3408, 2010.

[40] Z. Zuo et al., "Learning Contextual Dependence With Convolutional Hierarchical Recurrent Neural Networks," IEEE Trans. Image Process., vol. 25, no. 7, pp. 2983-2996, 2016.

[41] H. Li, Y. Li, and F. Porikli, "DeepTrack: Learning Discriminative Feature Representations by Convolutional Neural Networks for Visual Tracking," Br. Mach. Vis. Conf. 2014, pp. 1-12, 2014.

[42] P. Sermanet, K. Kavukcuoglu, S. Chintala, and Y. Lecun, "Pedestrian detection with unsupervised multi-stage feature learning," in Proceedings of the IEEE Computer Society Conference on Computer Vision and Pattern Recognition, 2013, pp. 3626-3633.

[43] B. Shuai, Z.Zuo, and G. Wang, "Quaddirectional 2D-Recurrent Neural Networks for Image
Labeling," IEEE Signal Process. Lett., vol. 22, no. 11, pp. 1990-1994, 2015.

[44] L. Deng, O. Abdel-Hamid, and D. Yu, "A deep convolutional neural network using heterogeneous pooling for trading acoustic invariance with phonetic confusion," in ICASSP, IEEE International Conference on Acoustics, Speech and Signal Processing - Proceedings, 2013, pp. 6669-6673.

[45] F. Alam, M. Imran, and F. Ofli, "Image4Act: Online social media image processing for disaster response," in Proceedings of the 2017 IEEE/ACM International Conference on Advances in Social Networks Analysis and Mining, ASONAM 2017, 2017, pp. 601-604.

[46] F. Alam, F. Ofli, and M. Imran, "CrisisMMD: Multimodal Twitter Datasets from Natural Disasters," May 2018.

[47] Y. Jia et al., "Caffe: Convolutional Architecture for Fast Feature Embedding," Jun. 2014.

[48] R. R Development Core Team, R: A Language and Environment for Statistical Computing, vol. 1, no. 2.11.1.2011.

[49] F. Chollet, "Keras Documentation," Keras.Io, 2015. [Online]. Available: https://keras.io/\%0Ahttps://keras.io.

[50] TensorFlow, "TensorBoard: Visualizing Learning," TensorFlow, 2017. [Online]. Available: https://www.tensorflow.org/ get_started/summaries_and_tensorboard.

[51] T. Geva, G. Oestreicher-singer, N. Efron, and Y. Shimshoni, "Using Forums and Search for Sales Prediction of High- Involvement Products," MIS $Q .$, vol. 41, no. 1, pp. 65-81, 2017.

[52] F. Cesarini, M. Lastri, S. Marinai, and G. Soda, "Encoding of modified X-Y trees for document classification," in Proceedings of the International Conference on Document Analysis and Recognition, ICDAR, 2001, vol. 2001-Janua, pp. 1131-1136.

[53] Y. Chen and H. Chen, "Opinion Spam Detection in Web Forum: A Real Case Study," Int. Conf. World Wide Web, no. 1, pp. 173-183, 2015.

[54] D. Zhang, L. Zhou, J. L. Kehoe, and I. Y. Kilic, "What Online Reviewer Behaviors Really Matter? Effects of Verbal and Nonverbal Behaviors on Detection of Fake Online Reviews," J. Manag. Inf. Syst., vol. 33, no. 2, pp. 456-481, 2016.

[55] K. Simonyan and A. Zisserman, "Very Deep Convolutional Networks for Large-Scale Image Recognition(VGGNet)," in ICLR 2015, 2015, pp. $1-14$.

[56] B. Li, X. Han, and D. Wu, "Real-Time Crowd Density Estimation Based on Convolutional Neural Networks," in 2018 International Conference on Intelligent Transportation, Big Data \& Smart City (ICITBS), 2018, pp. 690-694. 